\title{
Crowdsourcing to develop open-access learning resources on antimicrobial resistance
}

Eneyi E. Kpokiri ${ }^{1 * \dagger}$, Randall John ${ }^{2 \dagger}$, Dan Wu' ${ }^{1}$ Noah Fongwen' ${ }^{1}$, Jehan Z. Budak ${ }^{3}$, Christina C. Chang ${ }^{4}$, Jason J. Ong ${ }^{1,5}$ and Joseph D. Tucker ${ }^{1,6,7}$

\begin{abstract}
Objectives: Antimicrobial resistance (AMR) is a significant threat to global public health. Many medical curricula have limited clinical cases and materials focused on AMR, yet enhanced AMR education and training are needed to support antimicrobial stewardship programmes. We used crowdsourcing methods to develop open-access, learner-centred AMR resources. Crowdsourcing is the process of having a large group, including experts and non-experts, solve a problem and then share solutions with the public.

Methods: We organised a global crowdsourcing contest soliciting AMR-related multiple-choice questions, infographics, and images. First, we convened a diverse steering committee group to finalise a call for entries. Second, we launched the contest and disseminated the call for entries using social media, blog posts, email, and an in-person event. Partner institutions included two digital healthcare platforms: Figure $1^{\circledR}$ and Ding Xiang Yuan. Both organizations serve as online communities for healthcare specialists and professionals to report and comment on clinical information. At the end of the call, solicited entries were screened for eligibility and judged on merit and relevance to AMR learning and education. Exceptional entries were recognised, awarded prizes, and further reviewed for sharing with the public via open-access platforms.
\end{abstract}

Results: We received 59 entries from nine countries. These included 54 multiple-choice questions, four infographics, and one image. Eligible entries $(n=56)$ were reviewed and assigned a score on a 1-10 scale. Eight entries received mean scores greater than 6.0 and were selected as finalists. The eight finalist entries consisted of three infographics and five multiple-choice questions. They were disseminated through open-access publications and online medical communities. Although we launched a global call, we relied heavily on medical student groups and the entries received were not entirely globally representative.

Conclusions: We demonstrate that crowdsourcing challenge contests can be used to identify infectious disease teaching materials. Medical educators and curriculum developers can adapt this method to solicit additional teaching content for medical students.

Keywords: Antimicrobial resistance, Infectious diseases, Antimicrobial stewardship, Medical education, Curriculum development, Crowdsourcing

${ }^{*}$ Correspondence: eneyi.kpokiri@lshtm.ac.uk

${ }^{\dagger}$ Eneyi E. Kpokiri and Randall John contributed equally to this work

${ }^{1}$ Faculty of Infectious and Tropical Diseases, London School of Hygiene

and Tropical Medicine, Keppel St., Bloomsbury, London WC1E 7HT, UK

Full list of author information is available at the end of the article

\begin{abstract}
Background
Antimicrobial resistance (AMR) is a major global health problem. Antimicrobial stewardship programmes are increasingly designed to enhance and expand medical school infectious disease curricula [1-3]. Educational interventions have been shown to improve antimicrobial
\end{abstract}

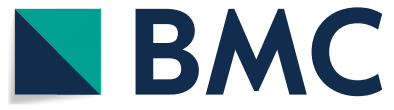

(c) The Author(s) 2021. Open Access This article is licensed under a Creative Commons Attribution 4.0 International License, which permits use, sharing, adaptation, distribution and reproduction in any medium or format, as long as you give appropriate credit to the original author(s) and the source, provide a link to the Creative Commons licence, and indicate if changes were made. The images or other third party material in this article are included in the article's Creative Commons licence, unless indicated otherwise in a credit line to the material. If material is not included in the article's Creative Commons licence and your intended use is not permitted by statutory regulation or exceeds the permitted use, you will need to obtain permission directly from the copyright holder. To view a copy of this licence, visit http://creativecommons.org/licenses/by/4.0/. The Creative Commons Public Domain Dedication waiver (http://creativecommons.org/publicdomain/zero/1.0/) applies to the data made available in this article, unless otherwise stated in a credit line to the data. 
use and practices $[4,5]$. Educational antimicrobial stewardship can be beneficial to clinicians in high and lowincome settings to increase their understanding of AMR. Studies from the United States and Europe suggest gaps in medical student exposure to appropriate antimicrobial prescribing practices and AMR [6, 7]. In addition, separate surveys in the Congo and Ethiopia suggest poor levels of AMR understanding among healthcare providers and students $[8,9]$. Other studies from the United Kingdom and Belgium also demonstrate that there are inconsistencies between antibiotic prescribing guidelines and clinician practices $[9,10]$. To address AMR, the World Health Organization (WHO) suggests implementing more vigorous educational models and training for healthcare providers [11]. In response, we sought to identify medical education resources on AMR using crowdsourcing methods. Crowdsourcing has a large group, including experts and non-experts, solve a problem and then share solutions with the public [12]. In the past, crowdsourcing has been used to expand existing medical curricula and develop flashcard study tools for preclinical education [13-15]. Additionally, researchers have successfully crowdsourced challenging, high quality, and complex multiple-choice questions (MCQs) from medical students [16].

There are several reasons why crowdsourcing is an effective approach to address medical education development and AMR. First, medical curricula development can be an arduous process for medical educators [17]. Crowdsourcing provides a structured mechanism to involve a large number of individuals in the process of curriculum development [17].

Second, crowdsourcing contests allow organizers to engage community members and raise public awareness [12]. Many groups have suggested that AMR public awareness and public engagement are crucial [18-20]. Third, crowdsourcing draws on open science principles that are increasingly important within medical training and research. Fourth, crowdsourcing can engage junior physicians and build out a pipeline of people interested in medical education.

The purpose of this study was to describe a crowdsourcing contest soliciting AMR infographics, MCQs, and images for medical teaching. We solicited MCQ and infographic submissions because 1) MCQs are preferred by some students as study tools, and 2) surveys among healthcare providers suggest a preference for communicating clinical information through infographics in comparison to conventional text reports $[16,21]$. Medical educators and curriculum developers can adopt this method in the future to create AMR-focused learning materials for educational and antimicrobial stewardship efforts.

\section{Methods}

The crowdsourcing contest design was based on the framework provided by the UNICEF/UNDP/World Bank/WHO Special Programme for Research and Training in Tropical Diseases (TDR) Practical Guide on Crowdsourcing in Health and Health Research [12]. The WHO framework provides a systematic approach to crowdsourcing within health contexts. Although this framework focuses on the application of crowdsourcing in public health settings more broadly, we specifically sought to assess its use in the area of medical education and training. According to the WHO model, crowdsourcing has six steps: selecting crowdsourcing as the methodology, convening a steering committee, engaging communities to participate, receiving and judging contributions, recognising finalists, and sharing solutions (Table 1).

\section{The open challenge contest}

The International Diagnostics Centre at the London School of Hygiene and Tropical Medicine and SESH (Social Entrepreneurship to Spur Health) organised this contest. The contest was officially launched in April 2019, and the call was open for two months. An open call for entries was provided on a contest website and promoted using the online medical learning platforms and other social media channels. We collaborated with two digital healthcare platforms in order to disseminate contest promotional materials: Figure $1^{\circledR}$ and Ding Xiang Yuan. Figure $1^{\circledR}$ is a Toronto-based digital platform that allows health professionals to share and comment on clinical cases [22]. Similarly, Ding Xiang Yuan is a Chinese digital platform that allows physicians to share medical information [23]. We selected these platforms for two reasons. First, the platforms allowed us to promote the contest in multiple languages (English and Chinese) and access potential participants in various geographical locations. Second, both platforms are specifically tailored for and used by clinicians and healthcare providers, which works well for our challenge contest as we sought to engage these particular groups to participate and send entries. After the open call was closed, all submitted entries were first screened for eligibility. Eligible entries were evaluated by three clinical experts who were identified by the steering committee and agreed to serve as contest judges. Each of the three clinical experts assigned entries a single score between 1 and 10 (with 1 denoting the weakest case, and 10 denoting exceptional submissions). The three scores were then averaged to determine a final single score for each entry. Eight entries that achieved a mean final score of 6.0 or greater emerged as finalists and were awarded a total of 1000 USD in gift cards. We selected 6.0 as a predetermined cut-off value to identify finalists 
Table 1 Stages employed in the contest

\section{Organising a steering committee}

The purpose of the steering committee was to provide guidance and outline an overall framework for contest execution. We convened a steering committee composed of ten individuals from five countries that met periodically through 60-min teleconference meetings over the duration of the contest to discuss design, organisation, and implementation. Members included experts in medicine, public health, infectious diseases, and medical education, as well as representatives from partner organisations who helped with contest promotion and dissemination

\section{Engaging the community to contribute}

The contest was officially launched on April 15, 2019. The website provided detailed information on the contest, including the purpose, categories of participation, rules, steering committee members, and partner organisations. In order to foster creativity and avoid cognitive fixation, we did not provide examples of entries. Promotional information was disseminated through social media (Instagram and Twitter), blog posts, email, and personal contacts. Emails were sent to relevant entities and individuals, including medical student interest groups at multiple institutions (such as interest groups in internal medicine, infectious diseases, and global public health), researchers, and medical specialists across multiple countries. We promoted the contest using Figure $1^{\circledR}$ and Ding Xiang Yuan (DXY). Both are digital platforms that bring together medical students, healthcare providers, and other healthcare-oriented professionals to share, distribute, and comment on medical cases. [18] DXY is China's largest online healthcare community, with more than four million registered users. On DXY's main platform website, we paid for four banner advertisements and one push notification to registered users, which reached 16,323 individuals and had 99 unique opens. We also created an official Figure ${ }^{\circledR}$ profile to facilitate contest promotion and developed a promotional infographic that was distributed via the Figure $1^{\circledR}$ app and made using Canva, an online graphic-design tool In order to encourage participation on Figure $1^{\circledR}$, we posted two MCQs on their platform. Each question focused on correct antibiotic treatment options based on presenting symptoms. As of November 9, 2019, the first promotional MCQ received 31 user-comments and 13,762 views and the second MCQ received 54 user-comments and 17,429 views. Promotion was also conducted through the official Figure ${ }^{\circledR}$ app email account Finally, we promoted the contest through one in-person event. Contest flyers were distributed at an AMR-focused conference in Belfast, United Kingdom where over 150 healthcare professionals working in the field of antimicrobial resistance and infection prevention and control convened We analysed metrics from both Figure $1^{\circledR}$ and the contest website. Figure $1^{\circledR}$ metrics showed that there were 85 comments, 107 saves, and 31,191 views from both promotional MCQs. Email analytics from the Figure $1^{\circledR}$ official email account suggest that details regarding the contest were disseminated to a high number of individuals. This first email had 764 opens, 491 unique opens, and 50 clicks. A digest email that consisted of both AMR contest and Figure $1^{\circledR}$ app content and was then sent to primary care physicians, nurses, and medical students. This email had 13,485 email opens, 8979 unique clicks, and 74 clicks on AMR contest content. A third email was sent on May 22, 2019 specifically asking Figure $1^{\circledR}$ users to contribute AMR related MCQs. This email had 19,298 opens, 12,243 unique opens, and 6781 clicks

Using online analytical tools, official contest website metrics were obtained between April 30 and June 9, for a total of 41 days. During this period, the website received 578 total clicks, for an average of 14.1 clicks per day, or 98.7 clicks per week. By reviewing IP addresses, it was determined that website clicks originated from 36 different countries

\section{Receiving and evaluating contributions}

Participants were given the option to submit their entries through a digital form made using Qualtrics ${ }^{\ominus}$ Survey Software (Qualtrics, Provo, UT) or upload their entry through either the Figure $1^{\circledR}$ app or DXY, provided that they previously had a platform account. Individuals could submit multiple entries. In addition to entries, we collected the following sociodemographic details regarding participants: name, institution, and country. We asked participants to specify a target audience for their entry (general and primary care physicians, medical students, pharmacists, infectious disease specialists, nurses, etc.) and identify any AMR learning objective(s) that were addressed, whether it be in regards to background, prevention, diagnosis, or treatment of AMR (Table 2). Consent was sought from participants regarding the modification and use of solicited entries for learning purposes, along with records of proper citation of external sources, and documentation of patient confidentiality Eligibility was determined based on pre-specified criteria: that the entry focused on AMR, was in English, and was in the correct format (as either a MCQ accompanied by answers, an infographic accompanied by brief explanatory captions, or an image). After determining eligibility, entries were transferred to evaluators for phase 1 judging. Evaluation was conducted by three clinical experts who were identified by the steering committee. Their participation as a judge was voluntary

During phase 1, each judge awarded every entry an individual score between 1 and 10 based on predetermined criteria. Predetermined evaluation criteria included adherence to the required format, contribution to existing learning resources, relevance and effectiveness in enhancing awareness and understanding of AMR, and focus on one or more of four AMR learning objectives (Table 2 ). The three individual scores were then averaged to determine one single score. Comments and revisions to further improve and develop finalist entries were sent to participants

\section{Recognising finalists}

The eight finalists with mean scores of 6.0 or greater were awarded a total of 1000 USD in prize money through Amazon gift cards and cash. Entries were awarded differing cash prize amounts based on the strength of the submission. All submitters were awarded a commendation certificate in recognition of their participation. The judges also received a thank you letter

\section{Sharing solutions}

After further review by expert judges, entries were shared with the public. The MCQs were arranged into a slide deck and included as an additional study material to an AMR learning module that was developed through a similar challenge contest in 2018 [18] Nine MCQs were selected by Figure $1^{\circledR}$ to be shared on their platform via posts, reaching a total of 68 comments, 126 saves, and 81,928 views. Three finalist infographics were published as posters on F1000Research, a life sciences-focused digital publishing platform

We asked participants to identify a target audience for their entry. Target audiences identified by submitters included medical students, general practitioners and physicians, internal medicine specialists, infectious disease specialists, microbiologists, prescribers in low- and middle-income countries, junior doctors, and nurses. Submitters also identified veterinary practitioners and farmers as a target audience due to the increasing prevalence of antibiotics in agriculture practices and raising livestock

as we deemed entries with an average score of $\geq 6.0$ to be of relatively high quality and value. After revising entries, the MCQs were arranged into a slide deck similar to an AMR learning module developed through a challenge contest [24]. The finalist infographics were published as posters on F1000Research, a life sciences-focused digital publishing platform [25-27]. We asked participants to specify the AMR learning objective(s) (Table 2) that their 
Table 2 AMR learning objectives

\begin{tabular}{|c|c|}
\hline Overall objectives & Individual objectives \\
\hline $\begin{array}{l}\text { Background } \\
\text { Information }\end{array}$ & $\begin{array}{l}\text { 1. Interpret local epidemiologic data or antibiograms to determine local rates of AMR infections } \\
\text { 2. List key risk factors for drug-resistant infections }\end{array}$ \\
\hline Prevention & $\begin{array}{l}\text { 3. Describe factors that may lead to unnecessary antibiotic prescribing by healthcare providers } \\
\text { 4. Describe the types of precautions needed/infection control measures for AMR organisms }\end{array}$ \\
\hline Diagnosis & $\begin{array}{l}\text { 5. Interpret susceptibility of testing results to select the most appropriate antibiotic regimen } \\
\text { 6. Utilise the local (and regional, if available) microbiology lab to help interpret patient test results }\end{array}$ \\
\hline Treatment & $\begin{array}{l}\text { 7. Identify infections that do not require antibiotic therapy } \\
\text { 8. Recognise that treatment of infections may require both antibiotic therapy and source control } \\
\text { 9. Recognise the concept of using the narrowest spectrum antibiotic for the shortest period of time } \\
\text { 10. Utilise a multidisciplinary healthcare approach when managing AMR organism } \\
\text { 11. List resources that can be useful in the treatment of patients with AMR infections } \\
\text { 12. Describe the incidence and spectrum of adverse antibiotic effects }\end{array}$ \\
\hline
\end{tabular}

entry addressed. Consensus on prioritizing AMR learning objectives were developed through a modified Delphi survey with stakeholders in AMR [28]. The Delphi survey was conducted amongst attendees in a one day AMR symposium that held in London, United Kingdom. These learning objectives also overlap with the Strategic Objectives outlined by the WHO's Global Action Plan on Antimicrobial Resistance [29]. The contest was organised in line with terms and conditions as specified by the legal committee of the London School of Hygiene and Tropical Medicine (LSHTM). As part of the conditions of the contest, participants were required to obtain informed consent from subjects where any personal data was included in the entry. Ethics approval was deemed unnecessary by the institutional review board at LSHTM.

\section{Results}

We received 59 entries with 56 eligible entries that came from nine countries: Cameroon $(n=30)$, the United States $(n=10)$, Nigeria $(n=5)$, the United King$\operatorname{dom}(\mathrm{n}=3)$, Australia $(\mathrm{n}=3)$, Jordan $(\mathrm{n}=2)$, Singapore $(n=1)$, India $(n=1)$, and China $(n=1)$. Of the 56 eligible entries, there were $51 \mathrm{MCQs}$, four infographics, and one image. Of the 56 eligible entries, 54 were solicited through the official contest website, and two were solicited through the online learning platforms.

The average score of all entries $(n=56)$ was 4.84. Breakdown of final scores by entry type shows that infographics $(n=4)$ had an average score of 7.00 , images $(n=1)$ had an average score of 5.00, and MCQs $(n=51)$ had an average score of 4.67 .

Finalist entries centred on a wide range of topics in AMR education and research. MCQs selected as finalists focused primarily on AMR background information and prevention/treatment. Topics included effective infection control in health institutions, multi-drug resistant organisms, antibiotic usage in animal farming, antibiotic treatment options in response to persistent symptoms, and mechanisms of AMR. Participants also identified veterinary practitioners and farmers as a target audience due to the increasing prevalence of antibiotics in agriculture practices and raising livestock [23]. The finalist infographics addressed AMR background information, treatment, and diagnosis. Finalist submissions are included in Additional file 1.

\section{Discussion}

We have demonstrated that crowdsourcing methods can be used to identify open-access medical education materials on antimicrobial resistance (AMR). Our findings support existing literature demonstrating that crowdsourcing is a feasible method to develop educational resources in the medical and public health fields. $[13,15$, 16] This challenge contest is a unique example of how to implement crowdsourcing methods to create medical education curricula specifically for the purposes of enhancing antimicrobial stewardship efforts.

This study draws on insights and examples from different settings. The contest was global in scope, as we received 56 eligible entries from nine different countries across five continents. We were able to solicit entries from both high-income and low-income countries, as well as entries from different practice areas, such as human medicine, veterinary medicine, and hospital- and community-based medicine. In addition, we were able to identify relevant online platforms to support contest implementation. The use of online platforms facilitated broad dissemination to an international audience and spurred engagement surrounding AMR and antibiotic prescribing practices. The inclusion of several social media metrics in our study also offers key insight into the use of digital platforms in crowdsourcing challenge contests and can guide future contest-organisers who wish 
to interact with online platforms for contest organisation and promotion.

This challenge contest received high-quality submissions, consistent with other crowdsourcing studies [30]. In our contest, eight entries (representing 14\% of all submissions) achieved a mean score of 6.0 or greater, which was similar to the frequency of high-quality submissions in another global health innovation contest on Hepatitis $B$ and C [30]. In terms of entry dissemination, three infographics were identified for online publication through F1000Research, nine MCQs were shared through the online learning platforms and $22 \mathrm{MCQs}$ were selected for inclusion in a study slide deck on AMR. 25 out of 56 eligible entries (representing $43 \%$ of the submissions) were selected for dissemination, which was slightly higher than the dissemination frequency from other challenge contests [30, 31].

Crowdsourcing has several advantages. First, through contest promotion, we were able to spur creativity and awareness surrounding AMR and acquired a diverse and global range of ideas. Soliciting MCQs, infographics, and images on AMR from medical students, physicians, and other healthcare professionals in multiple countries suggests that crowdsourcing is also feasible across different settings. Second, medical curricula development can be a time-consuming and challenging task for a small number of individuals $[13,17]$. We demonstrate that a bottom-up crowdsourcing approach can be a cost-effective method to develop medical teaching materials rapidly, decreasing the potential burden on educators and curriculum developers. This suggests that similar to other studies, crowdsourced materials from both experts and non-experts can be used in medical education [14, 15, 32]. Consistent with existing literature, our crowdsourcing approach involved coordinating with finalists in order to edit and refine submissions [13]. An important aspect of crowdsourcing is the process of having experts and non-experts work collaboratively in order to arrive at a final solution. Although the process of editing submissions can be more time-consuming, it also has some intrinsic value in terms of spurring engagement and participation across a wide continuum of stakeholders and participants. Future research should investigate methods to further optimize and streamline the process of developing medical education content through crowdsourcing.

Although many people viewed the contest promotion announcements on online platforms, we received few submissions from them: only two entries were submitted through the online platforms. This data suggests that paid online platforms to promote participation in challenge contests may be less effective, indicating a need for additional crowdsourcing interventions studying the use of paid online platforms. Given that in-person promotion of challenge contests has been associated with a greater volume of entries, more attention to in-person activities may also be useful for promotion [33, 34].

Our study has implications for research and policy surrounding medical education and curriculum development. While our study shows how crowdsourcing is an effective strategy to develop additional study resources in medical education, there is a need for more research to evaluate the impact and effectiveness of these educational resources. Robust programmes are essential in evaluating the extent to which the use of medical education stewardship approaches translate into improvements in clinical practice and understanding. There is also a need to review current curricula on AMR to identify content gaps and inform future projects.

Our contest had some limitations. First, we heavily targeted medical student groups. However, the timing of the call for entries overlapped with the examination calendar of many medical and public health schools, while others were already on break. Second, our participation may have been limited as we only promoted the contest at one in-person AMR event. Third, although our contest was global in scope, our entries were not entirely representative of all global settings, as there were no entries from the Latin American region. Fourth, entries accepted through the online learning platforms were limited to those in the English language and Chinese (Ding Xiang Yuan). This may have affected participation from non-English and non-Chinese speaking countries. Also, due to the small sample size, future studies and data are needed to establish crowdsourcing as an approach to address medical education and AMR training.

\section{Conclusions}

This study enhances our understanding of crowdsourcing in the context of medical education. Our contest demonstrates that crowdsourcing can be used to increase study materials available for medical students and physicians. Clinical educators could consider adopting crowdsourcing approaches to enhance medical education and mitigate traditional barriers associated with curriculum development. There is a need for additional research testing the impact and efficacy of crowdsourced clinical training resources for students and practitioners.

\section{Supplementary Information}

The online version contains supplementary material available at https://doi. org/10.1186/s12879-021-06628-0.

Additional file 1. Additional figures and tables. 


\section{Acknowledgements}

The authors wish to thank SESH (Social Entrepreneurship to Spur Health) for creating and hosting the contest website, and the online platforms (Figure $1^{\circledR}$ and Ding Xiang Yuan) for providing support in promoting the open call for entries. We also thank members of the steering committee for providing guidance at all stages of the contest and all the judges who volunteered to review submissions

\section{Authors' contributions}

JDT conceived the study design with input from JZB, CCC, and EEK. RJ, DW and NF facilitated promotion of the call. JZB, CCC, and JJO reviewed finalist entries. EEK and RJ prepared the results and drafted the manuscript. All authors read and approved the final manuscript.

\section{Funding}

This project was supported by the Academy of Medical Sciences and the Newton Fund (Grant Number NIF\R1\181020).

\section{Availability of data and materials}

Additional data collected from entries are available in additional files.

\section{Declarations}

\section{Ethics approval and consent to participate}

Not applicable. The institutional review board at the London School of Hygiene and Tropical Medicine deemed this work a non-human subject research and ethics was not necessary. All participants in the open call completed an informed consent statement at the point of submitting the entries.

\section{Consent for publication}

Not applicable. Where patients' medical records were used, informed consent was obtained by participants prior to submission and all identifying information has been removed.

\section{Competing interests}

The authors declare that they have no competing interests.

\section{Author details}

${ }^{1}$ Faculty of Infectious and Tropical Diseases, London School of Hygiene and Tropical Medicine, Keppel St., Bloomsbury, London WC1E 7HT, UK. ${ }^{2}$ Department of Health Policy and Management, Gillings School of Global Public Health, University of North Carolina, Chapel Hill, NC, USA. ${ }^{3}$ Department of Medicine, Division of Allergy \& Infectious Diseases, University of Washington, Seattle, WA, USA. ${ }^{4}$ Partners ID Images, Department of Infectious Diseases, Massachusetts General Hospital, Boston, MA, USA. ${ }^{5}$ Central Clinical School, Monash University, Melbourne, Australia. ${ }^{6}$ Social Entrepreneurship To Spur Health (SESH), Guangzhou, China. ${ }^{7}$ Institute of Global Health and Infectious Diseases, University of North Carolina, Chapel Hill, NC, USA.

Received: 12 November 2020 Accepted: 20 August 2021 Published online: 06 September 2021

\section{References}

1. Hsu JL. Building an antibiotic stewardship program: an interactive teaching module for medical students. MedEdPORTAL. 2018;14:1.

2. Silverberg SL, Zannella VE, Countryman D, et al. A review of antimicrobial stewardship training in medical education. Int J Med Educ. 2017;8:353.

3. Laks M, Guerra CM, Miraglia JL, Medeiros EA. Distance learning in antimicrobial stewardship: innovation in medical education. BMC Med Educ. 2019;19(1):1-9.

4. Kandeel A, Palms DL, Afifi S, et al. An educational intervention to promote appropriate antibiotic use for acute respiratory infections in a district in Egypt-pilot study. BMC Public Health. 2019;19(3):498.

5. Roque F, Herdeiro MT, Soares S, Rodrigues AT, Breitenfeld L, Figueiras A. Educational interventions to improve prescription and dispensing of antibiotics: a systematic review. BMC Public Health. 2014;14(1):1-20.
6. Abbo LM, Cosgrove SE, Pottinger PS, et al. Medical students' perceptions and knowledge about antimicrobial stewardship: how are we educating our future prescribers? Clin Infect Dis. 2013;57(5):631-8.

7. Dyar OJ, Pulcini C, Howard P, et al. European medical students: a first multicentre study of knowledge, attitudes and perceptions of antibiotic prescribing and antibiotic resistance. J Antimicrob Chemother. 2014;69(3):842-6.

8. Thriemer K, Katuala Y, Batoko B, et al. Antibiotic prescribing in DR Congo: a knowledge, attitude and practice survey among medical doctors and students. PloS ONE. 2013;8(2):e55495.

9. Abera B, Kibret M, Mulu W. Knowledge and beliefs on antimicrobial resistance among physicians and nurses in hospitals in Amhara Region Ethiopia. BMC Pharmacol Toxicol. 2014;15(1):1-7.

10. Mainjot A, D'Hoore W, Vanheusden A, Van Nieuwenhuysen JP. Antibiotic prescribing in dental practice in Belgium. Int Endod J. 2009;42(12):1112-7.

11. Organization WH. WHO competency framework for health workers' education and training on antimicrobial resistance. 2018.

12. Organization WH, UNICEF. Crowdsourcing in health and health research: a practical guide. 2018.

13. Blackwell KA, Travis MJ, Arbuckle MR, Ross DA. Crowdsourcing medical education. Med Educ. 2016;50(5):576.

14. Tackett $S$, Raymond M, Desai R, et al. Crowdsourcing for assessment items to support adaptive learning. Med Teach. 2018;40(8):838-41.

15. Bow HC, Dattilo JR, Jonas AM, Lehmann CU. A crowdsourcing model for creating preclinical medical education study tools. Acad Med. 2013:88(6):766-70.

16. Grainger R, Dai W, Osborne E, Kenwright D. Medical students create multiple-choice questions for learning in pathology education: a pilot study. BMC Med Educ. 2018;18(1):1-8.

17. Shappell E, Chan TM, Thoma B, et al. Crowdsourced curriculum development for online medical education. Cureus. 2017;9:12.

18. Redfern J, Bowater L, Crossley M, Verran J. Spreading the message of antimicrobial resistance: a detailed account of a successful public engagement event. FEMS Microbiol Lett. 2018;365(16):Fny175.

19. Redfern J, Bowater L, Coulthwaite L, Verran J. Raising awareness of antimicrobial resistance among the general public in the UK: the role of public engagement activities. JAC-Antimicrobial Resistance. 2020;2(1):dlaa012.

20. N W. The importance of public engagement on antibiotic resistance. . January 18, 2019. 2010. https://sangerinstitute.blog/2019/01/18/ the-importance-of-public-engagement-on-antibiotic-resistance/.

21. Turck CJ, Silva MA, Tremblay SR, Sachse SL. A preliminary study of health care professionals' preferences for infographics versus conventional abstracts for communicating the results of clinical research. J Contin Educ Heal Prof. 2014;34:S36-8.

22. 1 F. A digital platform for health professionals to share and comment on clinical cases. May 27, 2021,

23. R. L. Tencent backs Chinese healthcare portal DXY in $\$ 500 \mathrm{M}$ round. TechCrunch. 2020;

24. Kpokiri EE, Budak JZ, Chang CC, et al. Innovative strategies to fight antimicrobial resistance: crowdsourcing to expand medical training. F1000 Research. 2020;9(227):227

25. Khan SJR, Wu D, Kpokiri E. Carbapenem resistant gram negative CNS infections: threats to commonly used antimicrobials in treatment of MDR gram negatives. F1000 Research. 2020. https://doi.org/10.7490/ f1000research.1117824.1.

26. Khan SJR, Wu D, Kpokiri E. Ceftazidime-Avibactam-Aztreonam synergy testing: The use of combination ceftazadime-avibactam and aztreonam in the treatment of metallobetalactamse producing carbapenem resistant gram negative infections. F1000 Research. 2020. https:// doi.org/10.7490/f1000research.1117824.1.

27. Khan S JR, Wu D, Kpokiri E. Multidrug resistant fungal infections: challenges arising with treatment options. 2020; doi: https://doi.org/10. 7490/f1000research.1117825.1

28. Budak J, Kpokiri EE, Abdoler E, Tucker J, Schwartz B. 2547. Prioritizing Antimicrobial Resistance Learning Objectives Through a Modified, Two-Round, One-Day Delphi at a Multidisciplinary Conference. 2019:

29. Organization WH. Global action plan on antimicrobial resistance. 2015. 2019. 
30. Tucker JD, Meyers K, Best J, et al. The HepTestContest: a global innovation contest to identify approaches to hepatitis B and C testing. BMC Infect Dis. 2017;17(1):177-85.

31. Tang W, Wei C, Cao B, et al. Crowdsourcing to expand HIV testing among men who have sex with men in China: a closed cohort stepped wedge cluster randomized controlled Trial. PLoS Med. 2018;15(8):e1002645.

32. Dai JC, Lendvay TS, Sorensen MD. Crowdsourcing in surgical skills acquisition: a developing technology in surgical education. J Grad Med Educ 2017;9(6):697.

33. Zhang Y, Kim JA, Liu F, et al. Creative contributory contests (CCC) to spur innovation in sexual health: Two cases and a guide for implementation. Sex Transm Dis. 2015;42(11):625.
34. Zhang Y, Tang S, Li K, et al. Quantitative evaluation of an innovation contest to enhance a sexual health campaign in China. BMC Infect Dis. 2019;19(1):1-8

\section{Publisher's Note}

Springer Nature remains neutral with regard to jurisdictional claims in published maps and institutional affiliations.
Ready to submit your research? Choose BMC and benefit from:

- fast, convenient online submission

- thorough peer review by experienced researchers in your field

- rapid publication on acceptance

- support for research data, including large and complex data types

- gold Open Access which fosters wider collaboration and increased citations

- maximum visibility for your research: over $100 \mathrm{M}$ website views per year

At BMC, research is always in progress.

Learn more biomedcentral.com/submissions 